\title{
Costos directos de la hemodiálisis en unidades públicas y privadas
}

\author{
Luis Durán-Arenas, MC, PhD, (I) Paula D Ávila-Palomares, Lic en Econ,(I) Rodrigo Zendejas-Villanueva, Lic en Econ,(I) \\ María Magaly Vargas-Ruiz, CP, MASS,(I) Laura L Tirado-Gómez, MC, M en C, D en C, (1,2) \\ Malaquías López-Cervantes, MC, MPH, PhD.(I)
}

Durán-Arenas L, Ávila-Palomares PD, Zendejas-Villanueva R, Vargas-Ruiz MM, Tirado-Gómez LL, López-Cervantes M. Costos directos de la hemodiálisis en unidades públicas y privadas. Salud Publica Mex 20I I;53 supl 4:S5I6-S524.

\section{Resumen}

Objetivo. Estimar y comparar el costo por sesión de hemodiálisis dentro del sector público y privado en la Ciudad de México. Material y métodos. Se calcularon los costos de los insumos utilizados en las hemodiálisis de cuatro hospitales públicos y dos privados de la Ciudad de México, mediante la estrategia de micro-costeo denominada PAATI. Para la obtención de los datos se utilizó el método de sombra; además, se empleó Excel para elaborar cédulas en las que se determina el PAATI para cada sesión. Resultados. El costo anual promedio directo en el sector público por el tratamiento de un individuo en hemodiálisis es de $\$ 158964.00$ M. N., y el costo de atender a la población que podría demandar terapia de reemplazo renal se estima en \$10 92I 788072.00 M. N. Conclusión. La disponibilidad de recursos humanos e infraestructura en el país es muy limitada para el campo de la nefrología en general $y$, en particular, para ofrecer servicios de hemodiálisis, por lo que sería necesario inyectar más recursos para poder responder ante la demanda por insuficiencia renal terminal.

Palabras clave: unidades de hemodiálisis en hospital; costos y análisis de costos; costos de atención a la salud; economía de la salud; asignación de recursos; México
Durán-Arenas L, Ávila-Palomares PD, Zendejas-Villanueva R, Vargas-Ruiz MM, Tirado-Gómez LL, López-Cervantes M.

Direct cost analysis

of hemodialysis units.

Salud Publica Mex 201 I;53 suppl 4:S5 I6-S524.

\section{Abstract}

Objective. To estimate and compare direct costs per hemodialysis session in public and private units in Mexico City. Material and Methods. PAATI, a microcosting strategy, was used to determine total costs of four public and two private health hospitals in Mexico City. A "shadow study" approach was employed to collect the needed data. Charts containing the "PAATI" information for each session were developed in Microsoft Excel. Results. The average annual cost per patient undergoing hemodialysis in public units is $\$ 158964.00 \mathrm{MX}$. The estimated cost for the care of all population estimated in need of renal replacement therapy (via hemodialysis) was estimated to be $\$ 10921788072.00$ MX. Conclusion. Human resources and infrastructure availability in México are very limited for nephrology, and in consequence for offering hemodialysis services.

Key words: hemodialysis units; hospital; costs and cost analysis; health care costs; health economics; source allocation; Mexico

(I) Facultad de Medicina, Universidad Nacional Autónoma de México. México.

(2) Instituto Nacional de Pediatría. México.

Fecha de recibido: 30 de agosto de 2010 - Fecha de aceptado: 17 de junio de 2011

Autor de correspondencia: Dr. Malaquías López Cervantes. Unidad de Proyectos Especiales de Investigación, Facultad de Medicina.

Torre de Investigación, ler. piso. Ciudad Universitaria. 04510 Delegación Coyoacán, México, DF.

Correo electrónico: 
T a insuficiencia renal terminal es un problema cada Lvez más grave en México, tanto por la prevalencia cada vez mayor de diabetes e hipertensión, como por los problemas del manejo adecuado de estas enfermedades en los servicios de salud del país. En este número de la revista se presenta un análisis de la situación de esta enfermedad en México, por lo que no se ahondará en esta introducción; será suficiente decir que la terapia de reemplazo renal en sus diversas modalidades tienen ya una larga experiencia en el país. Paniagua y Amato, en su excelente revisión sobre este tema desde hace más de un lustro, han llamado la atención sobre los problemas que se enfrentan en la seguridad social. ${ }^{1}$ Sin embargo, son pocos los estudios que abordan este problema en la población no asegurada.

En la revisión de la bibliografía se identificaron 18 estudios realizados en distintas partes del mundo sobre los costos del tratamiento de reemplazo renal (TRR), ${ }^{2,3}$ en los que se consideraron las tres modalidades de TRR (trasplante renal, diálisis peritoneal y hemodiálisis) y sus diferentes particularidades y, en la mayoría de ellos, los costos fueron calculados anualmente.

En el presente trabajo, se enfoca únicamente en el análisis de la hemodiálisis, ya que en otro reporte ${ }^{4}$ se consideran otras opciones de TRR. En forma sucinta, la hemodiálisis tiene como objetivo principal depurar la sangre de los pacientes con enfermedad renal crónica (ERC) por medio de la circulación de la sangre del paciente con una máquina que emula la función del riñón y filtra los tóxicos. Por lo general, este procedimiento se realiza en hospitales o en unidades especializadas que requieren el equipo de filtración y purificación.

El hecho de que en el país, ya sea en hospitales $o$ en unidades separadas, se conformen unidades de hemodiálisis facilita definir con mayor factibilidad los costos directos asociados con la producción de sesiones de hemodiálisis. No obstante, las estimaciones de costos que se han publicado, por lo general, sólo consideran el expediente del paciente y no las unidades de hemodiálisis, o se basan en las declaraciones de los expertos, por lo que existe una gran variación de costos en la bibliografía internacional. Por ejemplo, los estudios que estimaron los costos del trasplante renal concluyeron que éste es el tratamiento más barato y el mejor al que pueden someterse los pacientes con ERC; sin embargo, la mayoría no consideró los costos a largo plazo de la terapia de inmunosupresión. ${ }^{5}$

Del mismo modo, la metodología utilizada en las investigaciones revisadas habitualmente se basa en opiniones de expertos y en la revisión de expedientes. ${ }^{6-8}$ Este tipo de revisiones, dada la limitación de los expedientes, tienden a ser poco profundas y no incluyen todos los aspectos relevantes del costeo, ya que dependen en gran medida de la calidad de los expedientes, la cual es con frecuencia cuestionable en países en vías de desarrollo.

Por lo anterior, el presente estudio tiene como objetivo estimar el costo por sesión de hemodiálisis en México dentro de los sectores público y privado, mediante la aplicación de una estrategia denominada PAATI, en unidades de hemodiálisis de la Ciudad de México., ${ }^{*}$

\section{Material y métodos}

El estudio se llevó a cabo durante el mes de octubre del año 2009 en seis distintas unidades de hemodiálisis de la Ciudad de México que se seleccionaron por conveniencia, una por cada institución de salud pública (Instituto Mexicano del Seguro Social -IMSS-, Instituto de Seguridad y Servicios Sociales de los Trabajadores del Estado-ISSSTEPetróleos Mexicanos -PEMEX- y Secretaría de Salud -SSA-) y dos unidades privadas. Con lo anterior, se buscó que las unidades fueran representativas de las diferentes instituciones de salud que existen en el país, además de ser unidades promedio en términos de volumen.

El cálculo de los costos de las sesiones de hemodiálisis se realizó mediante la aplicación de la técnica de PAATI (programa, acciones, actividades, tareas e insumos) desarrollada por uno de los autores de este artículo como una estrategia para la estimación de los costos de programas de salud pública. Esta metodología sigue los principios de la estimación de costos basada en actividades, pero se adapta a las características de los programas de salud. ${ }^{10}$

Para la obtención de los datos se utilizó la estrategia de estudio de sombra, que consiste en seguir paso a paso los movimientos y actividades que realiza el personal encargado de llevar a cabo los diferentes procedimientos dentro de una sesión de hemodiálisis. De esta manera, se registraron los tiempos, movimientos e insumos utilizados en las sesiones de hemodiálisis en seis unidades, cuatro del sector público y dos del sector privado (cuadro I). Durante las visitas, se observaron y cuantificaron el tiempo, material, cantidad y personal (médico nefrólogo, enfermera general o especialista y personal de intendencia) que formaban parte fundamental del desarrollo de la sesión.

Se empleó una hoja de cálculo de Excel para elaborar una cédula, en la que se capturaron los datos del PAATI para cada sesión de hemodiálisis, con el fin de establecer la unidad de medida, cantidad de recur-

\footnotetext{
* Durán-Arenas L. Metodología para la estimación de costos en programas de salud: programas, acciones, actividades, tareas e insumos (PAATI). Instituto Nacional de Salud Pública, 2000. [Documento no publicado].
} 


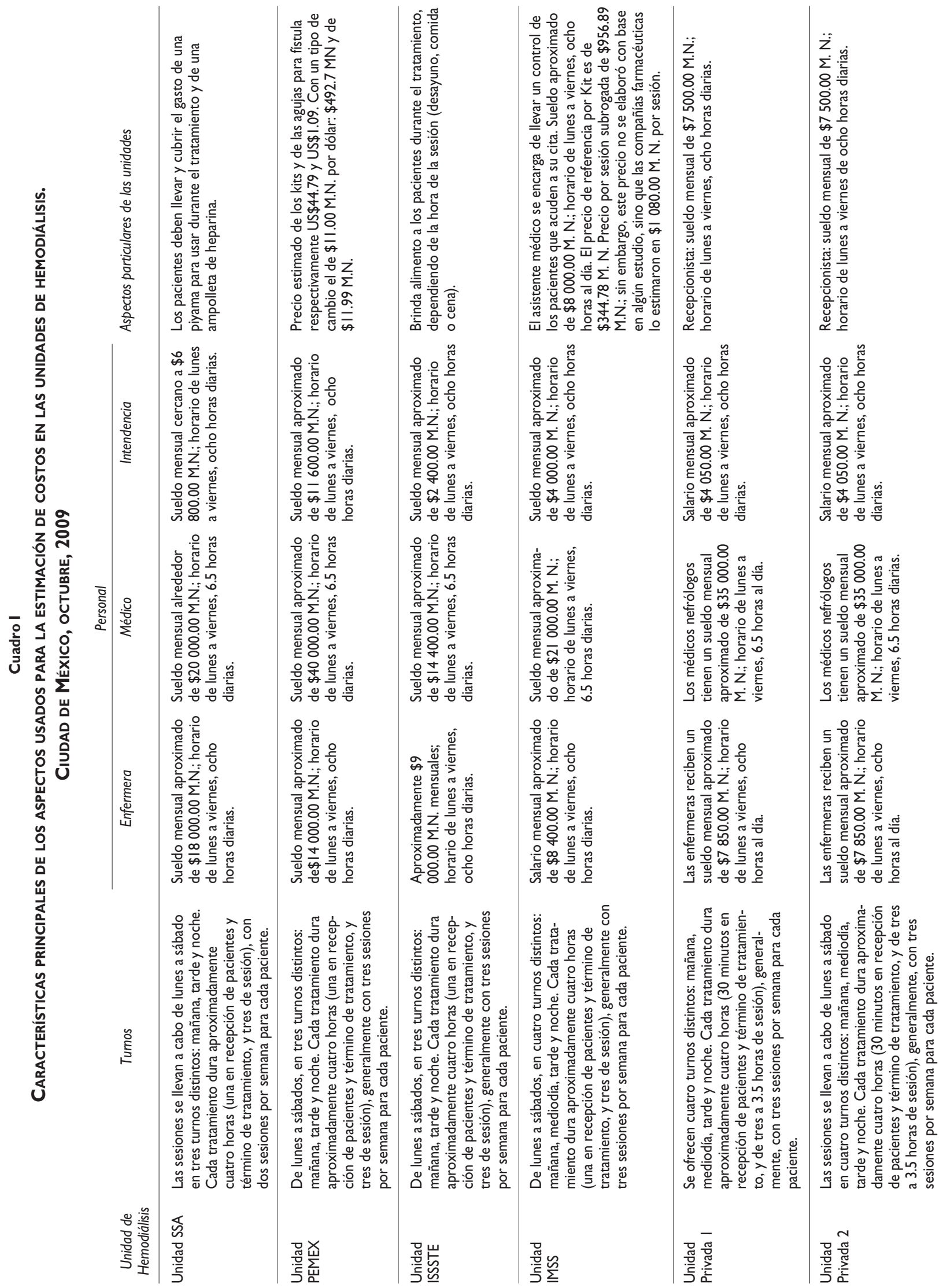


sos, costos unitarios y costos totales de los recursos empleados. El costo de los insumos utilizados para el desarrollo de las sesiones se obtuvo de dos formas: 1) Los insumos y reactivos utilizados directamente en el proceso de hemodiálisis se obtruvieron de las mismas unidades; 2) para el resto de insumos variables no específicos del proceso de filtrado se obtuvieron los costos por medio de cotización directa, con los precios para el público en general (al menudeo) y se clasificaron en dos grupos: médicos y no médicos. Los materiales médicos se cotizaron en la farmacia Lanceta, ${ }^{*}$ Doctor Solutions ${ }^{\ddagger}$ y Supplier Partnerships for Customer Solutions. ${ }^{\S}$ Los precios de los materiales no médicos se obtuvieron de la tienda de autoservicio Soriana.\#

Para el cálculo proporcional del costo del personal que interviene en las sesiones de hemodiálisis, se asumió con base en el juicio del grupo asesor de nefrólogos del proyecto que: los médicos laboran dentro de un horario de lunes a viernes (20 días por mes) con 6.5 horas de trabajo al día; las enfermeras y personal de intendencia también laboran de lunes a viernes, pero con turnos de ocho horas diarias; el salario que perciben los médicos, enfermeras y personal de intendencia se investigaron de manera directa, es decir, preguntándole al personal que se encontraba laborando en el momento de la visita; sólo en el caso de los hospitales privados la información fue proporcionada por la compañía farmacéutica que brinda el servicio, ya que es ésta quien los contrata y paga.

El tiempo promedio por sesión fue de tres horas, por lo que se estima que el consumo de agua tratada (por medio de filtro de carbón, suavizadores y ósmosis inversa) fue de 90 litros (30 litros/hora). Dado lo anterior, se estableció que el costo por litro de agua tratada es de $\$ 1.00 \mathrm{M}$. N., lo que implica un costo total de agua tratada por sesión de $\$ 90.00 \mathrm{M}$. N. A continuación se presentan los resultados del análisis comparativo de las unidades estudiadas (las estimaciones en detalle de cada uno de los PAATI por unidad de hemodiálisis se encuentran en el reporte final de la investigación). ${ }^{4}$ Se elaboró un modelo semanal y anual con el cual fue

\footnotetext{
* Lanceta HG [sitio de internet]. México: Lanceta HG, 2008. [consultado: 2008 noviembre 3]. Disponible en: http:/ / www.lancetahg.com.

₹ Doctor Solutions [sitio de internet]. México: Doctor Solutions, 2008. [consultado: 2008 noviembre 3]. Disponible en: http:// doctorsolutions.com.

$\S$ VWR [sitio de internet]. West Chester, PA: VWR International, LLC; 2009. [Actualizado: 2009 dic 10; consultado: 2008 noviembre 3]. Disponible en: https:/ / www.vwrsp.com.

\# Soriana [sitio de internet]. Monterrey: Tiendas Soriana, 2009. [consultado: 2008 noviembre 3] Disponible en: http:/ / www1.soriana.com.

\& En este caso y todos los siguientes, se hace referencia al salario líquido que reciben mensualmente, es decir, con prestaciones y antigüedad (en su caso) incluidos.
}

posible estimar los costos en el ámbito nacional y, con ellos, el presupuesto aproximado para lograr la cobertura universal bajo los siguientes supuestos:

- Los costos unitarios de las sesiones son una aproximación confiable de los costos nacionales.

- Los datos de la prevalencia de pacientes con necesidad de hemodiálisis se tomaron del estudio "Enfermedad renal crónica y su atención mediante tratamiento sustitutivo en México". ${ }^{4}$

- En el ámbito nacional, todas las unidades están construidas con un modelo similar al de las unidades visitadas.

- No existen variaciones regionales por costos de insumos y recursos humanos.

- La ampliación de la cobertura estaría dada a partir de los fondos del Seguro Popular, puesto que no se considera una ampliación para el presupuesto del Instituto Mexicano del Seguro Social (IMSS).

\section{Resultados}

Entre otros aspectos particulares de las unidades se encontró que, en todas aquellas visitadas, las sesiones se llevan a cabo de lunes a sábado. La diferencia radica en que tres de los cuatro hospitales del sector público sólo ofrecen tres turnos diarios, mientras que las unidades privadas, y la pública restante, otorgan el servicio de hemodiálisis en cuatro turnos diariamente: mañana, mediodía, tarde y noche.

Cada tratamiento dura aproximadamente cuatro horas en todas las unidades; pero existen diferencias en el tiempo que los pacientes están conectados al riñón artificial. Se descubrió que, por lo general, en las unidades privadas, el tiempo que les toma a los pacientes prepararse para su sesión es de 30 minutos, contando la espera en recepción. Por lo tanto el tiempo efectivo de hemodiálisis es de tres a tres horas y media, dependiendo de las necesidades del paciente. Sin embargo, en las unidades privadas, la recepción y conexión de un paciente toma media hora y los pacientes no reciben más de tres horas de tratamiento.

Resulta importante señalar que, en cada una de las unidades de hemodiálisis estudiadas, existen variaciones importantes en el costo de salarios, especialmente entre nefrólogos y el personal de enfermería (cuadro I). En las unidades privadas, el salario de los médicos fue mayor entre 40 y $59 \%$ que en las públicas, exceptuando la unidad de PEMEX que paga una cantidad considerablemente mayor que cualquier otra unidad. En el caso del salario de las enfermeras, la relación fue inversa, pues se encontró que, en las unidades públicas, con excepción del IMSS, el salario fue mayor; la diferencia 
más alta se encontró en la Secretaría de Salud, en donde las enfermeras recibían un salario $56.4 \%$ mayor que el de la unidad con los costos de enfermería más bajos.

El presente estudio arrojó variaciones en los costos del TRR para pacientes con ERC, mediante sesiones de hemodiálisis, en las distintas unidades, las cuales se deben fundamentalmente a los costos asociados con el personal de salud (cuadro II). Además, el costo de los insumos para llevar a cabo el tratamiento es el que ocupa la mayor cantidad de recursos monetarios por sesión y

\section{Cuadro II}

Estimaciones de tiempo y costo de los insumos por procedimiento. Ciudad de México, octubre, 2009

Unidad de Hemodiálisis

$$
\begin{array}{cc} 
& \text { Catéter Mahurkar } \\
\hline \text { Tiempo (min.) } & \text { Costo }(\$)
\end{array}
$$

\begin{tabular}{ll} 
Unidad SSA & 77.30 \\
\hline Unidad PEMEX & 68.15 \\
\hline Unidad ISSSTE & 61.15 \\
\hline Unidad IMSS & 57.50 \\
\hline Unidad privada I & 66.20 \\
\hline Unidad privada 2 & 55.25
\end{tabular}

\begin{tabular}{|c|c|}
\hline$\%$ & Tiempo \\
\hline 12.53 & 53.3 \\
\hline 9.46 & 57.3 \\
\hline 5.46 & 52 \\
\hline 6.34 & 61.0 \\
\hline 5.03 & 53.5 \\
\hline 4.20 & 47.2 \\
\hline
\end{tabular}

$\begin{array}{ll}77.30 & 145.91 \\ 68.15 & 99.25 \\ 61.15 & 56.71 \\ 57.50 & 52.13 \\ 66.20 & 54.24 \\ 55.25 & 45 .\end{array}$

\begin{tabular}{ll}
$145.9 \mid$ & 12.5 \\
99.25 & 9.4 \\
$56.7 \mid$ & 5.4 \\
52.13 & 6.3 \\
54.24 & 5.0 \\
45.17 & 4.2 \\
\hline
\end{tabular}

Fístula AV Costo $(\$)$

Tiempo enfermera

\begin{tabular}{|c|c|c|c|c|c|c|}
\hline Unidad SSA & 7.00 & 17.92 & 1.54 & 7.00 & 17.92 & 1.73 \\
\hline Unidad PEMEX & 5.40 & 27.65 & 2.63 & 5.40 & 27.65 & 2.98 \\
\hline Unidad ISSSTE & 7.00 & 12.92 & 1.24 & 7.00 & 12.92 & 1.36 \\
\hline Unidad IMSS & 8.40 & 23.31 & 2.83 & 8.40 & 23.31 & 3.12 \\
\hline Unidad privada I & 2.00 & 8.97 & 0.83 & 2.00 & 8.97 & 3.12 \\
\hline Unidad privada 2 & 5.00 & 22.44 & 2.08 & 5.00 & 22.44 & 2.22 \\
\hline
\end{tabular}

\begin{tabular}{ll}
98.41 & 9.52 \\
\hline 83.89 & 9.03 \\
\hline 48.79 & 5.15 \\
\hline 53.37 & 7.15 \\
\hline 44.90 & 7.15 \\
\hline 38.74 & 3.83
\end{tabular}

\begin{tabular}{|c|c|c|c|c|c|c|}
\hline Unidad SSA & - & 952.19 & 81.80 & - & 868.99 & 84.09 \\
\hline Unidad PEMEX & - & 868.71 & 82.77 & - & 763.48 & 82.18 \\
\hline Unidad ISSSTE & - & 926.25 & 89.12 & - & 842.18 & 88.90 \\
\hline Unidad IMSS & - & 703.49 & 85.49 & - & 625.42 & 83.83 \\
\hline Unidad privada I & - & 921.58 & 85.52 & - & 860.38 & 83.83 \\
\hline Unidad privada 2 & - & 921.58 & 85.64 & - & 863.38 & 85.36 \\
\hline
\end{tabular}

Tiempo médico

\begin{tabular}{|c|c|c|c|c|c|c|}
\hline Unidad SSA & 17.00 & 12.04 & 1.03 & 17.00 & 12.04 & 1.17 \\
\hline Unidad PEMEX & 15.00 & 18.00 & 1.71 & 15.00 & 18.00 & 1.94 \\
\hline Unidad ISSSTE & 30.00 & 7.50 & 0.72 & 30.00 & 7.50 & 0.79 \\
\hline Unidad IMSS & 18.20 & 7.94 & 0.97 & 18.20 & 7.94 & 1.06 \\
\hline Unidad privada I & 35.00 & 15.12 & 1.40 & 35.00 & 15.12 & 1.06 \\
\hline Unidad privada 2 & 21.00 & 9.22 & 0.86 & 21.00 & 9.22 & 0.91 \\
\hline
\end{tabular}

Tiempo otros

\begin{tabular}{|c|c|c|c|c|c|c|}
\hline Unidad SSA & - & 35.99 & 3.09 & - & 35.99 & 3.48 \\
\hline Unidad PEMEX & - & 35.99 & 3.43 & - & 35.99 & 3.87 \\
\hline Unidad ISSSTE & - & 35.99 & 3.46 & - & 35.99 & 3.80 \\
\hline Unidad IMSS & - & 35.99 & 4.37 & - & 35.99 & 4.82 \\
\hline Unidad privada I & - & 77.66 & 7.21 & - & 77.66 & 4.82 \\
\hline Unidad privada 2 & - & 77.66 & 7.22 & - & 77.66 & 7.68 \\
\hline \multicolumn{7}{|l|}{ Total } \\
\hline Unidad SSA & 101.30 & I 164.05 & 100.00 & 77.35 & 033.35 & 100.00 \\
\hline Unidad PEMEX & 88.55 & 1049.60 & 100.00 & 77.70 & 929.01 & 100.00 \\
\hline Unidad ISSSTE & 98.15 & I 039.37 & 100.00 & 78.10 & 947.38 & 100.00 \\
\hline Unidad IMSS & 84.10 & 822.86 & 100.00 & 88.00 & 746.03 & 100.00 \\
\hline Unidad privada I & 103.20 & 077.58 & 100.00 & 90.55 & I 007.03 & 100.00 \\
\hline Unidad privada 2 & 81.25 & I 076.06 & 100.00 & 73.25 & 1011.43 & 100.00 \\
\hline
\end{tabular}

Otros insumos 
parece ser muy homogéneo entre las unidades (82-89\%) (cuadro III). El costo de los insumos del tratamiento en todas las unidades es el dominante en los costos totales de producción de sesiones de hemodiálisis (figura 1).

Es posible observar que, para las sesiones en pacientes con catéter o con fístula, el menor costo se encuentra en la unidad de hemodiálisis del IMSS. Por otra parte, el mayor costo se observa en la unidad de hemodiálisis de la SSA. En el caso de las unidades privadas, los costos son iguales entre sí y muy semejantes a las otras dos unidades públicas (cuadro II). Resulta importante notar que el tratamiento con catéter es más costoso que el tratamiento con fístula (figura 1). Esto se debe, en la mayoría de los casos, a que:

- El tiempo que le dedica el personal de enfermería es mayor (en promedio 10 minutos más).

- Requieren más gasas y solución antiséptica, debido a la limpieza del área.

- Se necesita un número mayor de jeringas, con el fin de verificar la permeabilidad de los lúmenes.

- La cantidad de heparina utilizada es mayor, puesto que, al final de cada sesión, se introduce cierta cantidad en los lúmenes para evitar la formación de coágulos.

- Se requiere un parche ex profeso para cubrir el catéter y evitar su contaminación, así como, tapones para los lúmenes.

Cabe mencionar que, si se considerara la reutilización de los filtros, con la precaución de usarlo sólo en 10 ocasiones, el costo se reduce considerablemente, incluso sin considerar los insumos necesarios para llevar a cabo esta operación (cuadro III). Finalmente, en el cuadro IV se extiende la estimación de las sesiones a la estimación de los costos y precios por semana típica, y anuales para establecer el presupuesto necesario si se quisiera lograr una cobertura universal. ${ }^{*}$ En este cuadro podemos observar las estimaciones de costos y precios del TRR por ERC por semana (tres sesiones). Los costos estimados para lograr una cobertura de la totalidad de la población con necesidad de TRR en una semana típica varía desde \$394 019871.00 M. N., a precios promedio en instituciones públicas, hasta \$420 364080.00 M. N. a precios de contratación de subrogación.

Resulta importante notar que los precios y los costos por sesión son muy cercanos. Esto puede deberse a que un solo proveedor ofrece la mayoría de los

\footnotetext{
* Por cobertura universal se entiende la extensión de los servicios de hemodiálisis a todos los pacientes que en el momento actual no reciben ni hemodiálisis ni diálisis peritoneal (65 006).
}

insumos para la hemodiálisis en México, y los insumos de acuerdo con nuestra estimación se llevan hasta $85 \%$ del costo de la sesión. También el mercado de la subrogación está muy concentrado (hay poca oferta de servicios de hemodiálisis). El costo anual promedio en el sector público por el tratamiento de un individuo en hemodiálisis es de $\$ 158964.00$ M. N., 10 mil pesos menos que el costo de producción promedio en el sector privado y del precio anual de la subrogación. Para alcanzar la cobertura universal, mediante el crecimiento de las unidades públicas, se necesitaría invertir 10333 millones de pesos. Para alcanzar la cobertura universal en contratación de unidades subrogadas se necesitarían 10952 millones de pesos.

\section{Limitaciones del estudio}

Actualmente no se cuenta con datos acerca de los costos en los que incurren los estados de la República por concepto de tratamiento de pacientes con hemodiálisis, ni de las unidades que brindan este servicio en México. Por esta razón, fue necesario obtener las cifras de costos por medio de una muestra por conveniencia en unidades de hemodiálisis, localizadas en la Ciudad de México. En este estudio sólo se incluyen costos directos y algunos costos variables por lo que, para realizar un estudio más completo, sería necesario considerar costos de inversión, depreciación del capital e infraestructura, así como el efecto de la inflación de insumos para poder estimar con más precisión los costos reales.

Es importante notar que, dependiendo de la estrategia para incrementar el número de las unidades de hemodiálisis, los costos no incluidos pueden variar. A pesar de estas limitaciones, este artículo ofrece datos que son de utilidad en la planeación de los servicios de salud, como se establece a continuación en el apartado de conclusiones.

\section{Conclusiones}

Este análisis posibilitó estimar los costos unitarios directos de las sesiones de hemodiálisis en seis unidades de la Ciudad de México. Como se señaló, el menor costo directo unitario fue el del IMSS (\$822.86), aproximadamente 250 pesos menos por sesión que los costos observados en el resto de las unidades de hemodiálisis estudiadas. En esta unidad en particular, se hace manifiesto el efecto del costo del personal de salud que participa en los distintos procedimientos de la hemodiálisis. En este sentido, el costo más importante es el del personal médico, que corresponde a una reducción de $40 \%$ del costo en hospitales privados y casi de $60 \%$, si se compara con PEMEX. A pesar del impacto de la 

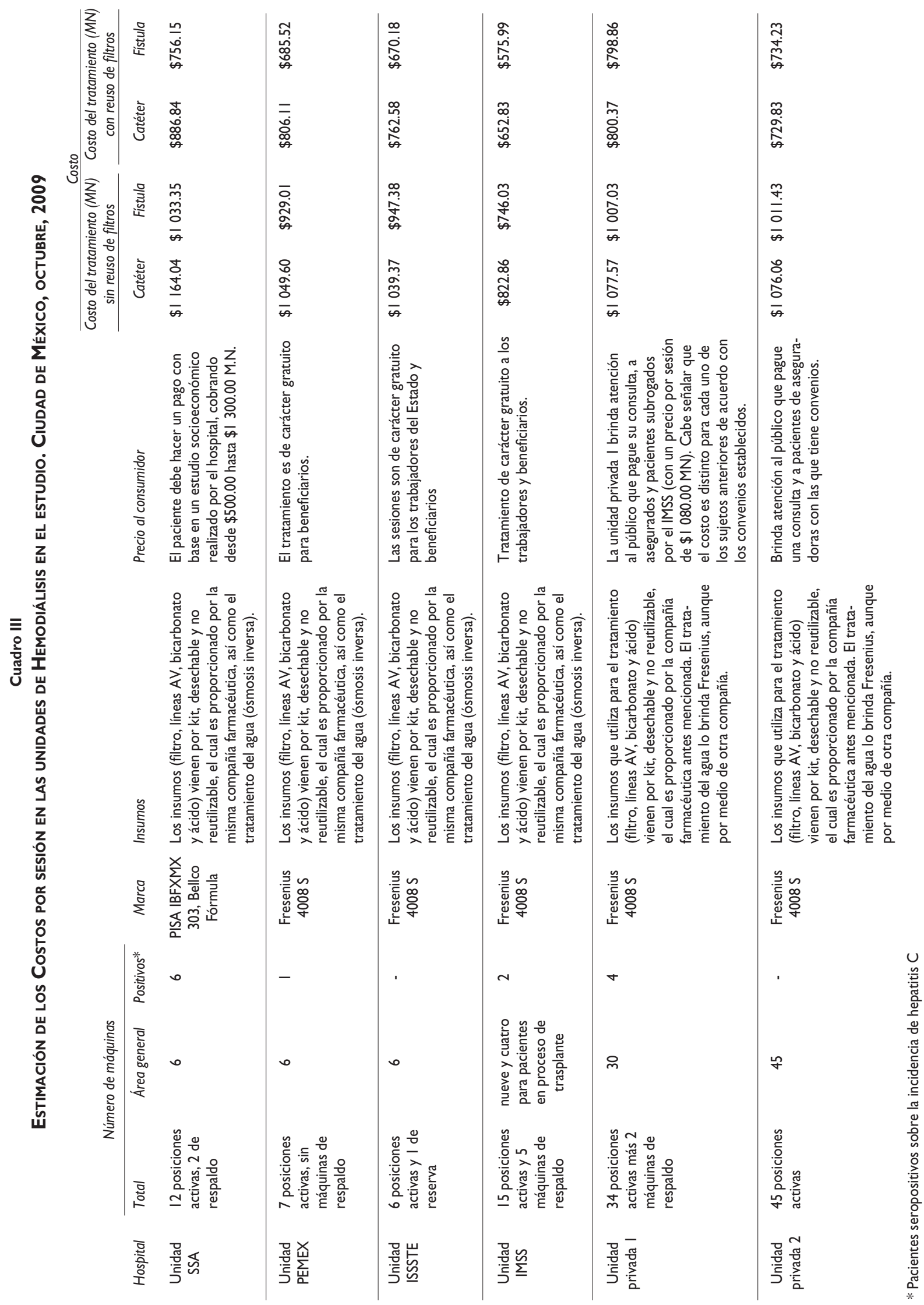


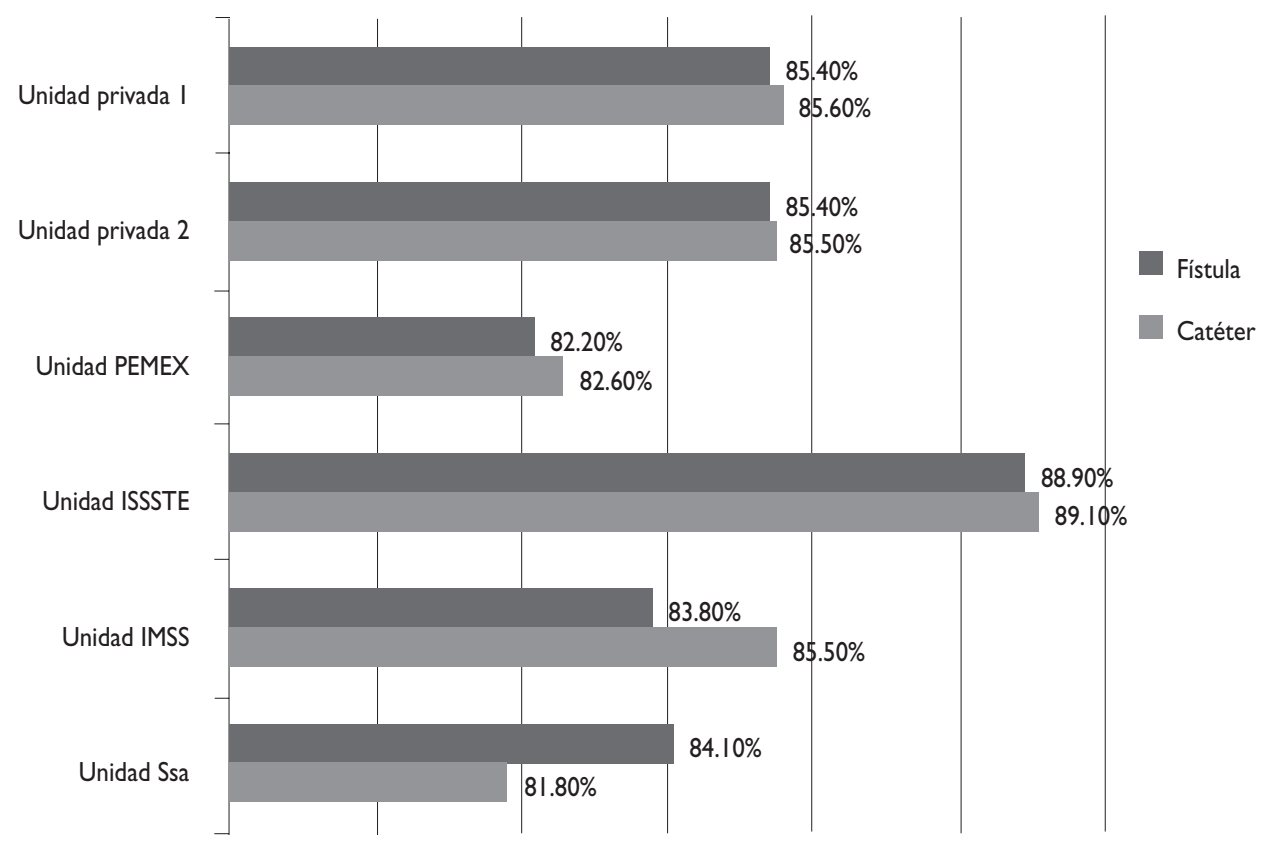

Figura I. Porcentaje del costo que corresponde a insumos de tratamiento por tipo de unidad de hemodiálisis. Ciudad de México, octubre, 2009

\section{CUADRO IV}

Estimación de costos totales de los pacientes con necesidad de TRR. Ciudad de México, octubre, 2009

Pacientes en hemodiálisis (19 097)

\begin{tabular}{llll} 
Costos estimados promedio por paciente en unidades públicas & $\$ 1019$ & $\$ 58379529$ & $\$ 3035735508$ \\
\hline Costos estimados promedio por paciente en unidades privadas & $\$ 1077$ & $\$ 61702407$ & $\$ 3208525164$ \\
\hline Precio de contratación por paciente IMSS & $\$ 1080$ & $\$ 61874280$ & $\$ 3217462560$
\end{tabular}

Pacientes sin tratamiento $(65006)$

Costos totales estimados promedio en unidades privadas

$\$ 168012$

$\$ 198723342$

$\$ 10333613784$

Costos totales estimados promedio en unidades públicas

$\$ 158964$

$\$ 210034386$

$\$ 10921788072$

Precio de contratación total anual por paciente IMSS

$\$ 168480$

$\$ 210619440$

$\$ 10952210880$

Pacientes en Diálisis Peritoneal 45639

De esta forma se alcanza el total de 129472 pacientes que requieren tratamiento de TRR

estructura de salarios, es importante notar que el resto de los ítems de costo son muy homogéneos entre las unidades de hemodiálisis. Como se presentó en la figura 1, entre 82 y $89 \%$ de los costos corresponden a insumos de tratamiento (que consideran todos los reactivos y materiales utilizados en las sesiones de hemodiálisis). Es importante reconocer que reutilizar el filtro puede tener implicaciones financieras importantes, por lo que en un análisis de costos posterior se ahondará en las implicaciones de política pública en el contexto del financiamiento para la hemodiálisis.

Utilizando estos costos unitarios como base y las estimaciones de prevalencia del estudio mayor fue posible calcular proyecciones del costo de ofrecer el servicio a 
afiliados al Seguro Popular en todo el país. ${ }^{4}$ Como se ha presentado en la sección de resultados, el costo de ofrecer una cobertura universal sería de más de 10000 millones de pesos, lo cual corresponde a casi $20 \%$ del presupuesto anual del Seguro Popular. No es intención del presente estudio señalar que el Seguro Popular debería de cubrir estos costos; sin embargo, las instituciones de seguridad social cubren, ya sea con hemodiálisis o diálisis peritoneal, a sus derechohabientes, lo que, en teoría, primordialmente haría recaer el esfuerzo en este programa.

Para el financiamiento de las intervenciones de salud y las discusiones sobre su monto y el establecimiento de prioridades, contar con estas estimaciones resulta esencial para enfrentar en forma más efectiva y eficiente los problemas de salud de la población en el país. Este tipo de estimación podría calcularse para las intervenciones principales del paquete de beneficios del Seguro Popular y, con base en ello, establecer criterios económicos comparativos para la definición de prioridades y la toma de decisiones.

Diferentes autores han declarado que el dinero es necesario, pero no suficiente para lograr la cobertura universal. ${ }^{11}$ En este documento se ha estimado un modelo ideal que, en la realidad, dependerá de la disponibilidad de recursos humanos e infraestructura del país; pero un primer paso es dimensionar el problema al que se enfrenta el país. En el estudio mayor, del que se deriva este trabajo, se ha dejado en claro la magnitud epidemiológica del problema de la insuficiencia renal crónica; en este trabajo se presenta la magnitud económica de una modalidad de tratamiento de la TRR. La imagen es de un alto impacto económico que plantea un reto para los tomadores de decisiones: ¿cómo lograr justicia distributiva para los miles de pacientes que requieren de TRR?

La situación del campo de la nefrología, en general, y en particular para ofrecer servicios de hemodiálisis, es muy limitada. En otra de las publicaciones derivadas del estudio mayor y publicadas en este número de la revista, se sugiere la necesidad de desarrollar una política de formación de nefrólogos en el país. De acuerdo con las estimaciones, se ha asumido que existe suficiente personal para ofrecer los servicios de nefrología; sin embargo esa no es la realidad: se tiene una escasez de nefrólogos, lo que se convierte en una barrera para la implantación de un programa que logre tener cobertura universal. Finalmente, el reto radica, entonces, en ampliar la aplicación de estudios económicos de este tipo que permitan visualizar los costos de las intervenciones a las que tiene que hacer frente el sistema de salud. Lo anterior, incluyendo consideraciones como que, para financiar alguna intervención, el SPSS requiere -por Ley General de Salud y su reglamento en materia de protección social en salud- un costeo basado en el protocolo técnico-clínico, avalado por el Consejo de Salubridad General, y un análisis actuarial de suficiencia presupuestal. De esta manera, se tendrá una base más sólida para la definición de presupuestos que consideren tanto los gastos operativos, como la necesaria inversión en infraestructura y recursos humanos.

Declaración de conflicto de intereses: Los autores declararon no tener conflicto de intereses.

\section{Referencias}

I. Paniagua R, Ramos A, Fabian R, Lagunas J, Amato D. Chronic kidney disease and dialysis in Mexico. Perit Dial Int 2007;27:405-409.

2. Just PM, Riella MC, Tschosik EA, Noe LL, Bhattacharyya SK, de Charro FT. Economic evaluations of dialysis treatment modalities. Health Pol 2008;86:163-180.

3. Kontodimopoulos N, Niakas D. An estimate of lifelong costs and QALYs in renal replacement therapy based on patients' life expectancy. Health Pol 2008;86:85-96.

4. López-Cervantes M, Rojas-Russell ME, Tirado-Gómez LL, Durán-Arenas L, Pacheco-Domínguez RL, Venado-Estrada AA, et al. Enfermedad renal crónica y su atención mediante tratamiento sustitutivo en México. México: Facultad de Medicina, Universidad Nacional Autónoma de México, 2009.

5. Croxson BE, Ashton T. A cost effectiveness analysis of the treatment of end stage renal failure. N Z Med J 1990;103:171-174.

6. Baboolal K, McEwan P, Sondhi S, Spiewanowski P, Wechowski J, Wilson $K$. The cost of renal dialysis in a UK setting, a multicentre study. Nephrol Dial Transplant 2008;23:1982-1989.

7. Hooi LS, Lim TO, Goh A, Wong HS, Tan CC, Ahmad G, et al. Economic evaluation of centre haemodialysis and continuous ambulatory peritoneal dialysis in Ministry of Health hospitals, Malaysia. Nephrology 2005; 10:25-32. 8. Arredondo A, Rangel R, de Icaza E. Costo-efectividad de intervenciones para enfermedad renal crónica terminal. Rev Saúde Pública 1998;32:556-565. 9. Betanzos-Reyes AF, Rodríguez MH, Durán-Arenas LG, Hernández-Ávila JE, Méndez-Galván JF, Velázquez-Monroy OJ, et al. Comparative analysis of two alternative models for epidemiological surveillance in the Mexican malaria control program. Health Policy 2007;80:465-482.

10. Hicks D. El sistema de costos basado en actividades ABC. México: Alfaomega, 2006.

II. Hsiao W. C. Why is a systematic view of Health financing necessary. Health Affairs. 2007;26(4):950-96I. 